\title{
Können Versicherungen zur großen Transformation beitragen?
}

\author{
Ist nicht die Gewinnmaximierung schuld an der Ausbeutung von \\ Mensch und Planet? Und ist nicht der Finanzsektor der Inbegriff \\ der Gewinnmaximierung? Der Artikel zeigt Ansatzpunkte auf, \\ mit denen der Finanzsektor zur Transformation beitragen kann. \\ Von Marie-Luise Meinhold
}

n den 1980er- und 1990er-Jahren dominierten zahlreiche Umweltverbrechen von Firmen die Schlagzeilen. Es stellt sich unweigerlich die Frage: Warum haben diese Firmen das überhaupt zugelassen? Die Antwort war schnell gefunden: Es liegt an der Gewinnmaximierung. Die Firmen stellen ihren Gewinn über das Wohl der Natur und der Menschen.

\section{Wo bleibt die Moral?}

Da entstand der Eindruck, die Wirtschaft sei irgendwie böse. Und der Finanzsektor, in dem gar nichts Materielles produziert oder verkauft wird, in dem es nur um Gewinnmaximierung geht, konnte in dieser Logik nur der Inbegriff des Bösen sein. Gewinnmaximierung in Reinform war bei mir und bei vielen anderen Menschen sehr verpönt. Wo bleibt da die Moral, das Gewissen?

Dabei habe ich die gestalterische Kraft des Finanzsektors unterschätzt, die kürzlich das EU-Chapter des Club of Rome um Bernard Lieater so gut herausgearbeitet hat (Lietaer 2013). Denn schon zu Zeiten meiner Doktorarbeit, ich beschäftigte mich damals unter anderem mit Bodensanierung, fiel mir auf, dass vor allem diejenigen Altlasten saniert wurden, bei denen die Reinigungsmaßnahmen über die Gewässerschadenhaftpflichtversicherung bezahlt wurden. Da wurde für mich erstmals erlebbar, dass es einen direkten Zusammenhang zwischen dem Boden, dem Planeten und dem abstrakten Finanzsektor gibt. Versicherungen sorgten dafür, dass Altlas- ten saniert wurden, weil sie die Mittel dafür bereitstellten.

Intuitiv traf ich damals auch die Entscheidung, diesen Bereich nicht länger abzulehnen. Mir war klar, dass in der Versicherungswirtschaft viel Geld bewegt wird, und dass dieses Geld wiederum Macht besitzt, und diese Macht kann viel bewegen, auch viel Gutes. Doch wie können mit dem Geld der Versicherungen positive soziale und ökologische Wirkungen erzielt werden? Dazu müssen Versicherungen, zusätzlich zur Rendite, systematisch berücksichtigen, welche Effekte sie im sozialen und im ökologischen Bereich haben.

Beispielsweise kann bei vergleichbaren Renditeparametern eine Investition in erneuerbare Energien $\mathrm{CO}_{2}$ einsparen, in Kindergärten kann sie Kindergartenplätze schaffen, in Mehrgenerationenprojekten kann sie Wohnplätze und Lebensraum schaffen. Das kann man sogar quantifizieren. Genauso können Investitionen in herkömmliche Kraftwerke $\mathrm{CO}_{2}$ freisetzen, das ist bei vielen produzierenden Gewerben der Fall, wenn sie nicht mit erneuerbaren Energien betrieben werden. So hat zum Beispiel Adelphi Consult schon 2010 nachgewiesen, welche Wirkungen Investitionen auf die $\mathrm{CO}_{2}$-Emissionen und damit auf den Klimawandel haben (Adelphi Consult 2010).

\section{Eine Finanzwende ist nötig}

Vor diesem Hintergrund ist eine Finanzwende nötig. Wir können nicht nur mit unserer Kaufkraft, auch mit unse- ren Anlageentscheidungen die Transformation mitgestalten. Wir sollten den Finanzinstituten den Vorzug geben, die diese Gestaltungskraft im Sinn der großen Transformation nutzen. Und noch etwas können Versicherungen tun. Sie können im Schadenfall Anreize für einen nachhaltigen Ersatz bieten. Sie können auch Kunden belohnen, die sich bereits besonders verantwortungsbewusst zeigen. Diese beiden Aspekte sollen kurz erläutert werden.

Wenn ein Sachschaden entsteht und etwas ohnehin kaputt ist, so ist der Versicherungsanspruch in der Sachversicherung in der Regel der nach gleicher Art und Güte, in der Haftpflichtversicherung in der Regel nach dem Zeitwert. Versicherungen können Tarife anbieten, die es den Kunden ermöglichen, beim Ersatz auch auf ökologische und soziale Aspekte zu achten. Kunden können sich beispielsweise für energieeffiziente Geräte entscheiden, für schadstofffreie Materialien oder für eine Herstellung unter fairen Arbeitsbedingungen, vielleicht sogar von Betrieben, die sich in besonderer Weise für soziale Belange einsetzen.

\section{Anreize für nachhaltigen Konsum}

Die Alltagserfahrung zeigt, dass solche ökologisch-fairen Produkte und Dienstleistungen in der Regel etwas teurer sind als die vergleichbaren Angebote, die ohne diese Belange auskommen. Versicherungen können den Kunden eine höhere Entschädigung in Aussicht stellen, wenn sie ökologische und soziale Aspekte bei der Beschaffung berücksichtigen.

Kunden, die besonders verantwortungsbewusst sind, achten in der Regel sehr auf sich und ihre Umgebung. Sie werden daher vermutlich weniger Schäden verursachen, weil sie mehr aufpassen. Das gilt insbesondere für Firmenkunden, die sehr umsichtig in ihren Prozessen aufgestellt sind und sich in hohem Maß für Recycling oder Wiederverwertung einsetzen. Durch diese besondere Sorgfalt entstehen auch weniger 
Schäden als bei Kunden, die beispielsweise mit Lösungsmitteln weniger sorgsam umgehen, wodurch es dort leichter zu Entflammungen kommen kann. Dieses Verantwortungsbewusstsein können Versicherer belohnen, indem sie diesen Kunden einen Preisnachlass gewähren, quasi als Vertrauensvorschuss.

\section{Versicherungen als Katalysatoren für die Vernetzung}

Schließlich haben Versicherungen die Möglichkeit, verschiedene Akteure zu vernetzen und so zur großen Transformation beizutragen. Das war ja eine der Kernaussagen des Wissenschaftlichen Beirats für Globale Umweltveränderungen zur Großen Transformation, dass eben verschiedene Gruppen zusammenkommen müssen, auch solche, die sonst wenig miteinander zu tun haben (WBGU 2011). Denn sie versichern oft Privatkunden und Firmenkunden gleichermaßen, sogar die Zivilgesellschaft mit ihren Stiftungen, Vereinen und Verbänden. Diese Vernetzung von verschiedenen Akteuren kann sogar große Vorteile für die Beteiligten bringen.

Die verantwortungsvollen Privatpersonen müssen oft hohe Suchkosten aufwenden, um die Firmen zu finden, deren Angebote ihren Bedürfnissen in so- zialer und ökologischer Hinsicht besser entsprechen. Genauso tun sich verantwortungsvolle Firmen schwer, die Privatpersonen zu erreichen, die sich für ihre öko-fairen Produkte interessieren. Solche verantwortungsvollen Firmen haben somit höhere Transaktionskosten als Firmen, die ohne besondere sozial-ökologische Belange direkt einen Massenmarkt ansprechen. Versicherungen, die einen Beitrag zur nachhaltigen Entwicklung leisten wollen, können als Kristallisationspunkte verantwortungsvolle Privatpersonen und Firmen vernetzen und so die Transaktionskosten für die Beteiligten senken.

\section{Ansätze für nachhaltige Versicherungen}

Vielleicht gelingt es sogar, dass auch die verantwortungsvollen Firmen Belohnungssysteme für ihre verantwortungsvollen Kunden einsetzen. Dann könnte die Vernetzung noch schneller in Gang kommen. Die größere Vernetzung würde wieder größere Netzwerkeffekte bringen und auf diese Weise könnte sich diese nachhaltige Wirtschaftsweise schneller durchsetzen und verbreiten.

Über die Ablehnung von schlechten Angeboten hinaus, wie es in den Konsumentenboykotten der Fall ist, können wir nicht nur zum strategischen Kon- sum kommen, sondern auch zur strategischen Empfehlung - und so im Konsum und im Finanzsektor die Wende hin zur großen Transformation schaffen.

\section{Literatur \\ Adelphi Consult (2010): Klimaeffekte von Kapital- anlagen - Eine Analyse der direkten und indirekten Wirkungen von Anlageprodukten - Zwischenbericht - Im Auftrag des Bundes- umweltministeriums. Berlin, Adelphi Consult. Lietaer, B. et al. (2013): Geld und Nachhaltig- keit - Von einem überholten Finanzsystem zu einem monetären Ökosystem. Ein Bericht des Club of Rome. München. \\ WBGU, Wissenschaftlicher Beirat für Globale Umweltveränderungen (2011): Welt im Wandel: Gesellschaftsvertrag für eine Große Transformation. Hauptgutachten 2011. Berlin.}

AUTORIN + KONTAKT

Dr. Marie-Luise Meinhold ist Vorstandsmitglied der Stiftung Nachhaltiges Leben und gründet mit ver.de die erste Sachversicherung, deren Ziel es ist, einen Beitrag zur nachhaltigen Entwicklung zu leisten.

Marie-Luise Meinhold, c/o Impact HUB, Gotzinger Str. 8, 81371 München. Tel.: +4989 2155-2480,

E-Mail: kontakt@unser-ver.de, Internet: www.unser-ver.de
Nachhaltigkeit

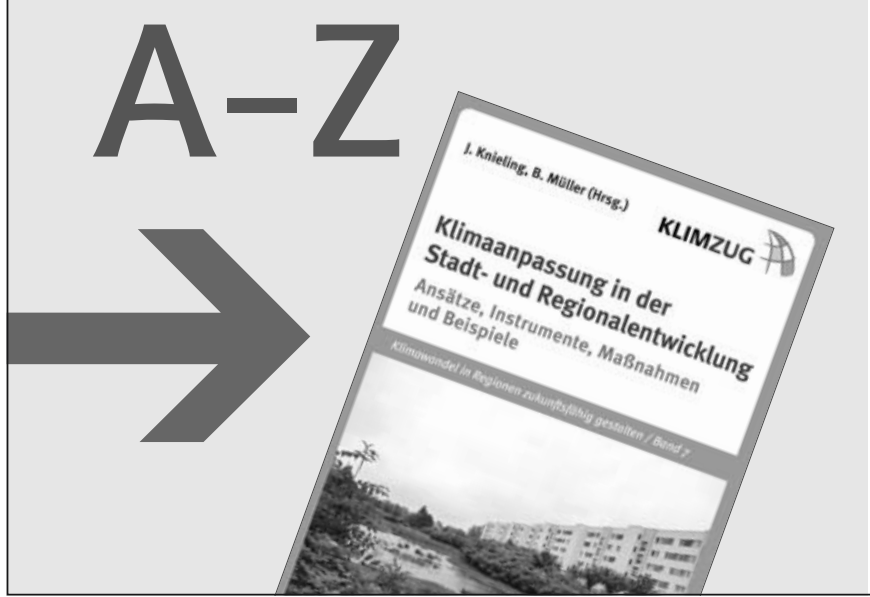

$W_{\text {wie Wetterextreme }}$

Während die internationale Politik ein weiteres Mal über Klimaschutzziele debattiert, sind die Folgen des Klimawandels bereits spür- und sichtbar. Ob Starkregen oder Dürren, ob Hitzerekorde oder Kältewellen - unsere Städte und Regionen müssen sich auf die sich wandelnden Rahmenbedingungen vorbereiten. Praxisansätze, Instrumente, Maßnahmen und Beispiele zeigen, wie den Herausforderungen des Klimawandels vor Ort zu begegnen ist.

J. Knieling, B. Müller (Hrsg.)

Klimaanpassung in der Stadt- und Regionalentwicklung

Ansätze, Instrumente, Maßnahmen

und Beispiele

464 Seiten, broschiert, 39,95 Euro, ISBN 978-3-86581-703-7

Erhältlich bei

www.oekom.de

oekom@verlegerdienst.de

Die guten Seiten der Zukunft 